\title{
Kirchliche Medienarbeit im Spannungsfeld des säkularen Rechts
}

von Peter Storr

\section{Problemstellung}

Kirchliche Medienarbeit bedient sich der säkularen Medien zur Erfüllung des kirchlichen Sendungsauftrages. Sie kann dabei sehr leicht in das Spannungsfeld des säkularen Rechts geraten.

1. Der Leiter der Abteilung Kirchenfunk einer deutschen Rundfunkanstalt verfaßte einen Kommentar über einen Konzilsfilm. Er warf der namentlich genannten Filmverleihfirma vor, "fix und clever“ auf den Tod des Konzilspapstes Johannes reagiert zu haben, weil der Filmtitel zu hohe und falsche Erwartungen erwecke. Der begleitende Text sei „banal geschwätzig“; Originalton und untermalende Musik seien „ein Graus“ für sich.

Dem Text sei die Fixigkeit anzuhören, mit dem der Film zweieinhalb Wochen nach dem Tode des Konzilspapstes für das deutsche Geschäft präpariert wurde.

Die Filmverleihfirma machte Schadensersatzansprüche geltend. Sie habe durch den Kommentar erheblichen Schaden erlitten, denn die Besucherzahl sei danach schlagartig zurückgegangen. Sie meinte, ihr seien unlautere Motive für die Aufführung des Films unterstellt worden, besonders die Wendung, sie habe "fix und clever" gehandelt, habe bei den Rundfunkhörern den Eindruck erweckt, der Film sei überstürzt auf den deutschen Markt geworfen worden, um aus der allgemeinen menschlichen Anteilnahme am Tod des Papstes Kapital zu schlagen.

2. Im Pfarrbrief seiner Gemeinde berichtete ein Pfarrer, ein namentlich genanntes Mitglied seiner Gemeinde habe eine große Spende geleistet. Er fuhr fort: „Ich mußte ihm sagen, daß er durch keine noch so große Spende den Schaden, den er angerichtet, wiedergutmachen kann. Es gilt das Wort: Man kann nicht Gott dienen und dem Mammon. Herr ... könnte mit seinen Kompagnons ..., der berüchtigten Frau Uhse und dem ... Verlag, der Pornoflut ein Ende bereiten. “ Der betreffende Spender fühlt sich bloßgestellt, insbesondere durch die den Tatsachen nicht entsprechende Behauptung, er arbeite mit der Pornoherstellerin Beate Uhse zusammen.

3. In einer kirchlichen Zeitschrift will kurz vor Kommunalwahlen eine linksgerichtete Wählergemeinschaft ein Wahlinserat aufgeben. Überschrift: „Christus würde $\mathrm{X}$ wählen.“

Unter Berufung auf die Pressefreiheit und Tendenzschutz lehnt die Zeitschrift das Inserat ab. Die Wählergemeinschaft beruft sich demgegenüber auf Chancengleichheit und Kontrahierungszwang.

4. In einer Kirchenzeitung erscheint ein Wahlkampfinserat einer den Kirchen nahestehenden Wählerinitiative mit folgenden Passagen: ... „Wählt keine Nationaldemokraten ... Die ersten Beulen einer neuen braunen Pest ... So wie heute begann es

Dr. iur. can. Peter Storr ist Rechtsanwalt in München und Professor für Recht an der Gesamthochschule Eichstätt im Fachhochschulstudiengang Sozialwesen. 
auch vor 35 Jahren in Deutschland ... Denkt an die grauenhaften Folgen des Tausendjährigen Reichs..."

Die von dem Text angesprochene NPD verlangte die Veröffentlichung folgender Gegendarstellung: Die Nationaldemokraten sind nicht die ersten Beulen einer neuen braunen Pest. Sie sind nicht verantwortlich für das, was vor 35 Jahren in Deutschland begann, noch für die grauenhaften Folgen des Tausendjährigen Reichs.

Diese Fälle - keineswegs konstruiert, sondern in leicht abgewandelter Form der Rechtssprechung oder der Praxis des Verfassers entnommen - werfen die Frage auf, ob die Grundrechte der freien Meinungsäußerung und der freien Religionsausübung der kirchlichen Medienarbeit Besonderheiten einräumen.

\section{Der Medienbegriff}

\section{Definition}

Zunächst stellt sich die Frage nach dem rechtlichen Begriff der Medien. Der Oberbegriff der Medien stellt eine Sammelbezeichnung für die sich an die breite Masse wendenden Mittel zur Verbreitung und Wiedergabe von Geistesgut dar. Der Inhalt und die geistige Höhe des wiedergegebenen Geistesgutes ist dabei gleichgültig. Gleichgültig ist aber auch die Art der Wiedergabe. Zum Medium im rechtlichen Sinn gehört also die Presse ebenso wie Funk, Film und Fernsehen. Auch der zahlenmäßige Umfang des Adressatenkreises bringt rechtlich keinen Unterschied. Das Pfarrblatt stellt genauso ein Medium dar wie der Kirchenfunk, der ein ganzes Gebiet der Bundesrepublik abdeckt, oder wie das "Wort zum Sonntag“ alle Fernsehteilnehmer der Bundesrepublik erreicht.

Auch die äußere Form und Gestaltung bringt keine rechtliche Differenzierung. Es ist gleichgültig, ob es sich um ein gedrucktes, festgebundenes Journal handelt oder eventuell nur ein hektographiertes oder im Umdruckverfahren hergestelltes Blatt wie den Pfarrbrief. Manche Landespressegesetze stellten dies ausdrücklich klar¹.

\section{Verfassungsrechtliche Grundlage}

Das jeweilige Medienunternehmen wird in seiner funktionellen Stellung und Eigenständigkeit gegen hoheitliche Eingriffe als Institution verfassungsrechtlich gewährleistet. So hat der Bundesgerichtshof z. B. in seinem Urteil vom 18.12.1968² festgestellt, daß Artikel 5 Grundgesetz als subjektives Recht zumindest in Verbindung mit anderen Grundrechten (bei uns kommt das Grundrecht der Freiheit der Religionsausübung in Frage) auch dic Freiheit des Verlegers oder Herausgebers einer Zeitung bzw. des Unternehmensinhabers eines anderen Mediums umfaßt, die in seinem Medium enthaltenen Informationen und Meinungen frei zu verbreiten und an möglichst viele Adressaten heranzubringen, daß ferner im Zweifel die Vermutung zugunsten einer weitgehenden Entfaltungsfreiheit streitet und daß gesetzliche Generalklauseln, die diese Freiheit begrenzen könnten, im Lichte jener grundsätzlichen Freiheitsrechte auszulegen sind. Das Bundesverfassungsgericht bezeichnet das Grundrecht auf freie Meinungsäußerung als schlechthin konstituierend für eine freiheitliche demokratische Staatsordnung ${ }^{3}$ und leitet hieraus das Grundrecht der Pressefreiheit $a b^{4}$. Dies beruht auf der Erkenntnis, daß in der freiheitlichen Demokratie nur die ständige geistige Auseinandersetzung zwischen den einander begegnenden 
sozialen Kräften und Interessen der wichtigste Weg zur Bildung des Staatswillens ist $^{5}$ und $\mathrm{da}$ sich diese Auseinandersetzung notwendig pluralistisch im Widerstreit verschiedener und aus verschiedenen Motiven vertretenen Auffassungen zu vollziehen hat ${ }^{\mathbf{6}}$. Dieser Meinungskampf ist nur durch Meinungs- und Pressefreiheit möglich. Eine derartige konstituierende Bedeutung für das demokratische Staatswesen gewinnt die Meinungs- und Pressefreiheit aber nur dann, wenn man sie nicht nur als individuelles Recht eines einzelnen betrachtet, sondern zugleich als „institutionelle“ Gewährleistung, die den Bestand eines freien und funktionsfähigen Presse- und Medienwesens als unerläßliche Voraussetzung des Meinungskampfes schützt ${ }^{7}$.

Aus dieser Institutionengarantie folgt, daß die Einrichtungen des Kirchenfunks, der Kirchenzeitungen und auch der Pfarrbriefe institutionell verfassungsrechtlich garantiert sind.

\section{Die Meinungsfreiheit als individualrechtliche Garantie}

\section{Allgemeiner Inbalt}

Die individualrechtliche Garantie der Meinungsäußerung und damit der Presse-, Funk- und Filmfreiheit erstreckt sich auf die Grundlagen für eine freie Meinungs-

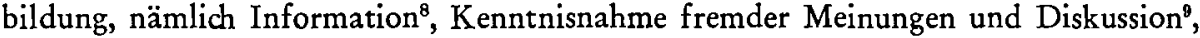
sowie die Äußerung und Verbreitung fremder Meinungen ${ }^{10}$.

Hierzu gehört ferner die eigene Denktätigkeit und das eigene Wollen bei der Meinungsbildung und schließlich die Außerung und Verbreitung der eigenen Meinung, einschließlich ihrer Wirkung auf Dritte ${ }^{11}$.

\section{Die Schranken der Meinungs- und Pressefreibeit}

Die Rechtsordnung der Bundesrepublik Deutschland schützt eine plurale Gesellschaft, das heißt, sie nimmt nicht einseitig eine Weltanschauung von ihrem Schutz aus und bevorzugt andererseits auch keine. Beim Schutz der Meinungsäußerungsfreiheit muß also notwendigerweise differenziert werden gegen Herabsetzung, Beleidigung, Mißachtung der Persönlichkeit oder des beruflichen und geschäftlichen Fortkommens anderer, sowie gegen Diskriminierung. Diese Einschränkung liegt im Wesen des Grundsatzes der Meinungsäußerungsfreiheit, denn, so führt das Bundesverfassungsgericht in ständiger Rechtssprechung aus, die Vermutung streitet für die Zulässigkeit der „freien Rede“, wenn es sich um einen Beitrag zum geistigen Meinungskampf in einer die Offentlichkeit wesentlich berührenden Frage durch einen dazu Legitimierten handelt. Um die freie Diskussion gemeinschaftswichtiger Fragen zu sichern, kann es nach den Umständen des Einzelfalles geboten sein, den Schutz privater Rechtsgüter zurücktreten zu lassen. Gerade in Auseinandersetzungen, die über einzelpersönliche Bezüge hinausgehen und eine Thematik von großer Tragweite für das Gemeinschaftsleben ansprechen, erfordert die Bedeutung des Art. 5 Grundgesetz, daß auch in der Art der Meinungsäußerung von Rechts wegen große Freiheit gewährt wird. Es ist deshalb zwischen Tatsachenbehauptungen und Werturteilen streng zu scheiden. Ein Rechtsschutz gegen Meinungsäußerungen ist besonders dann einzuschränken, wenn es darum geht, einen Angriff auf die von einem Kritiker vertretene Auffassung abzuwehren, den er aus seiner Sicht nach Tendenz und Aufmachung als unangemessen oder anstößig empfindet ${ }^{12}$. Diese Einschränkung ist dem Grundrecht 
der freien Meinungsäußerung und damit der Medienfreiheit bereits wesensimmanent $^{13}$. Insbesondere ergibt sich eine solche Abgrenzung aus den Art. 1 und 2 Grundgesetz und dem daraus abgeleiteten allgemeinen Persönlichkeitsrecht des Betroffenen. Weiterhin findet jedoch die Freiheit der Medienorgane ihre Grenzen an den allgemeinen Gesetzen, wie es Art. 5 Abs. 2 Grundgesetz ausdrückt. Darunter sind zu verstehen die Gesetze der allgemeinen Rechtsordnung ohne irgendwelchen Sondercharakter ${ }^{14}$. Es gehört hierzu also die gesamte allgemeine Rechtsordnung, der gegenüber Presse, Funk und Film keine Sonderstellung beanspruchen können. Für die urheberrechtliche Nutzung fremder Werke, das Wettbewerbsrecht und das Kartellrecht ergibt sich dies von selbst. Aus Art. 3 Grundgesetz sind hier keine Einschränkungen erkennbar. Dies gilt insbesondere, wenn es um Personen im natürlichen Sinne geht.

\section{Das allgemeine Persönlichkeitsrecht im Besonderen}

Rechtssprechung und Literatur haben nämlich nunmehr übereinstimmend anerkannt, daß die natürliche Person ein aus ihrem Personsein herausgewachsenes ursprïngliches, einheitliches, höchstpersönliches und daher unvererbliches, unübertragbares und unverzichtbares allgemeines Persönlichkeitsrecht hat. Dies gilt vor allem für folgende Bereiche:

a) Die Achtung als Mensch und auch die Achtung, die durch die individuelle Leistung und Lebensführung erworben ist. Es handelt sich hierbei um einen ergänzenden Schutz der persönlichen Ehre, die sich gegen unvollständige, verfälschende und unrichtige Darstellung des Lebens- und Persönlichkeitsbildes wehrt.

b) Zum allgemeinen Persönlichkeitsrecht gehört ferner die sog. Geheimsphäre, nämlich die geheimgehaltenen persönlichen Angelegenheiten wie Briefe oder sonstige private Aufzeichnungen, ferner

c) das abgeschlossene private Leben des Einzelnen und seiner Familie mit dem Schutz gegen Veröffentlichungen über das Privatleben und Familienangelegenheiten des Einzelnen, und schließlich

d) der Schutz der allgemeinen geistigen Entfaltung, Bewegungsfreiheit, der den Schutz des beruflichen und gewerblichen Bereichs ergänzt. Er richtet sich vor allem gegen heimliche Tonbandaufnahmen.

Die vorliegende Studie soll keine abschließende Darlegung der rechtlichen Schranken der kirchlichen Medienarbeit beinhalten.

\section{Lösung der Problemfälle}

Nach diesen wesentlichen und kurzen Ausführungen über den Kern der Beschränkung der Meinungsäußerung sollen die unter I dargestellten Fälle beleudhtet werden.

1. Die Werturteile, mögen sie auch noch so hart sein, waren nicht Gegenstand der richterlichen Uberprüfung. Auch ihre Richtigkeit oder Unrichtigkeit wurde nicht geprüft. Geprüft wurde lediglich, ob die Namensnennung des Filmverleihs zum Schadensersatz verpflichtet. Der Bundesgerichtshof stellte hierzu fest, daß es schon an der Ursächlichkeit für den Schadenseintritt fehle, weil die Namensnennung keine wesentliche Beeinträchtigung brachte ${ }^{15}$. Diese Entscheidung hat Raimer ${ }^{16}$ kritisiert, weil der einheitliche Vorgang, nämlich der gesendete Kommentar als Ganzes in einzelne Bestandteile zerlegt wurde. Diese Kritik halten wir nicht für gerechtfertigt, 
da die Kausalität in der Tat zu prüfen ist. In diesem Fall hätte es keinen Unterschied gemacht, ob der Name des Verleihers bekanntgegeben wurde oder nicht.

2. Der Fall des Pfarrbriefes ist schwieriger zu beurteilen. Im vorliegenden Fall durfte der namentlich Genannte schon deshalb nicht gebracht werden, weil die Tatsachenbehauptung (Zusammenarbeit mit Beate Uhse) falsch war. Aber auch wenn dies richtig gewesen wäre, muß man einen Eingriff in das allgemeine Persönlichkeitsrecht bejahen, weil die von ihm erbrachte Spende in Zusammenhang mit seiner beruflichen Tätigkeit gebracht wurde. Die Passage klingt zu sehr nach Freikauf von einer bewußten moralischen Verfehlung und bringt die individuelle Leistung und das Geschäftsgebahren eines namentlich Genannten in Verruf. Der Fall wurde gerichtlich nicht entschieden. Wir halten diese Art der Äußerung für gesetzlich nicht zulässig, abgesehen davon, daß sich der betreffende Pfarrer auch nicht in ein gutes Lidht setzt, wenn er eine Spende in nicht unbeträchtlicher Höhe annimmt und seinen Spender dann öffentlich disqualifiziert.

3. Anzeigengeschäfte der Presse werden auf der Grundlage privatrechtlicher Verträge abgewickelt. Für sie gilt der Grundsatz der Vertragsfreiheit, der die Abschlußfreiheit mitumfaßt. Das Bundesverfassungsgericht hat festgestellt, daß es im freien Belieben des Verlages steht, die Annahme gewisser ihm nicht genehmer Anzeigen abzulehnen ${ }^{17}$. Dies gilt grundsätzlich auch für politische Anzeigen, eingeschlossen Wahlanzeigen. Oft wurde bezweifelt, und zwar unter dem Gesichtspunkt der Chancengleichheit der Parteien, ob dies Rechtens sei. Es gibt immer wieder Stimmen, die das bezweifeln. Vor allem für das Rundfunkrecht gibt es sogar Entscheidungen, die dagegen sprechen ${ }^{18}$. Nun sind Rundfunkanstalten aber öffentlich-rechtlich organisiert, während Presseorgane zumeist privatrechtlich strukturiert sind. Problematisch wird diese Frage für den von uns angesprochenen Personenkreis eigentlich nur, wenn das kirchlich beherrschte Blatt gegenüber einer freien Wählergemeinschaft im lokalen Bereich fast konkurrenzlos ist, d.h., wenn das kirchliche Blatt eine Monopolstellung dahingehend genießt, daß in dem bestimmten Bezirk der anzusprechende Personenkreis anders nicht erreichbar ist.

So ist es feststehende Lehre und Rechtssprechung, daß an einem Ort, an dem es nur einen Bäcker gibt, dieser verpflichtet ist, seine Brötchen auch Atheisten zu verkaufen, weil bei dieser Monopolstellung auch im Privatrecht ein Kontrahierungszwang besteht.

Bei der Veröffentlichung von Meinungsäußerungen muß jedoch auch in diesem Extremfall die Institutionengarantie und hier das Recht auf freie Religionsausübung gewährleistet sein mit der Folge, daß ein kirchlich orientiertes Blatt unabhängig davon, ob es sich in privater oder in kirchlicher Hand befindet, nicht verpflichtet werden kann, tendenzwidrige Anzeigen zu veröffentlichen.

4. Hierzu ist grundsätzlich festzustellen, daß ein presserechtlicher Gegendarstellungsanspruch, wie er im Fall 4 beansprucht wurde, auch gegenüber kirchlichen Medien in Anspruch genommen werden kann. Nach heute allgemein herrschender Lehre und Rechtssprechung ist es dabei gleichgültig, ob sich der beanstandete Text im redaktionellen Teil eines Mediums befindet, oder sich als bezahlter Beitrag, insbesondere als Anzeige, darstellt. Entscheidend ist in diesem Fall darauf abzustellen, daß alle Pressegesetze $^{10}$ auf die Veröffentlichung einer Tatsachenbehauptung abstellen. Die Rechtssprechung hat hierzu den Leitsatz entwickelt: „Danach sind gegendarstellungsfähig 
Behauptungen über solche Tatsachen, die mit den Sinnen wahrnehmbar sind, sog. äußere Tatsachen. Ob wegen des formellen Verfahrens der Gegendarstellung, in dem über die Gewichtigkeit der Tatsachenbehauptung Beweis nicht erhoben werden kann, auch innere Tatsachen gegendarstellungsfähig sind, erscheint zweifelhaft, weil die Grenze zwischen Behauptungen über innere Tatsachen, wie beispielsweise politische Ziele und Meinungsäußerungen, insbesondere im politischen Kampf flüssig und daher für den Leser schwer feststellbar ist, was Tatsachenbehauptung und was politische Meinung ist. ${ }^{\text {20 }}$

Der Strafsenat des Bundesgerichtshofs hat wiederholt die Ansicht vertreten, daß bei politischen Auseinandersetzungen der Rahmen zugunsten der politischen Meinung weit zu ziehen sei, da das politische Geschehen anders als Tatsachenbehauptungen sich im allgemeinen einer gerichtlichen Nachprüfung entzieht ${ }^{21}$. Der Bundesgerichtshof hat in einer Entscheidung ${ }^{22}$ die Ansicht vertreten, daß die Außerung eines Kommunisten in einem Flugblatt, „der Bundeskanzler bereitet den Weltkrieg vor" und „der Bundeskanzler will die deutsche Einheit nicht", politische Werturteile seien. Selbst ein Tatsachenkern könne in diesen Äußerungen nicht festgestellt werden, die weltanschauliche Einstellung eines Mitteilers sei für die Auslegung seiner Außerungen von Bedeutung. Der Bundesgerichtshof ${ }^{23}$ hat ferner in dem Vorwurf, eine Person strebe nach einem gewissen Regierungsposten, nicht eine Tatsachenbehauptung erblickt. Logisch richtig stellt der BGH fest, ein Tatsachenkern liege jedem Werturteil zu Grunde. Wenn er aber nur unbestimmt angedeutet sei, so handle es sich nur um ein Werturteil.

Jedes Werturteil und jede Meinungsäußerung basiert auf Tatsachen, so wie sie der Außernde aufgenommen und innerlich verarbeitet hat. Eine politische Außerung ist das Ergebnis von Beobachtungen von äußeren Tatsachen und die Bekundung der eigenen inneren Stellungnahme hierzu.

Im Fall 4 kam das Oberlandesgericht Hamburg ${ }^{24}$ deshalb zu dem Ergebnis, daß es sich hier um Meinungsäußerungen handelt und nicht um Tatsachenbehauptungen, welch letztere allein gegendarstellungsfähig sind.

Daraus folgt der Schluß, daß auf Tatsachen basierende Meinungsäußerungen, wenn sie weltanschaulich motiviert sind, von der Rechtssprechung auf dem Umweg der Definition als sog. "innere Tatsachen" anders behandelt werden als weltanschaulich neutrale.

\section{Zusammenfassung und Ergebnis}

Die kirchliche Medienarbeit wird im säkularen Recht nicht anders behandelt als jede andere Medienarbeit. Es gelten für sie genauso die Schranken des allgemeinen Rechts wie für jede andere Medieninstitution. Allein bei der rechtlich relevanten Unterscheidung von Tatsachenbehauptung und Werturteil ist von der weltanschaulichen Wertung her ein weiterer Spielraum gegeben. Dies beruht jedoch nicht auf Art. 4 Grundgesetz, sondern nach herrschender Rechtssprechung auf der Tatsache, daß jedes Werturteil und jede Meinungsäußerung innerlich auf Tatsachen zurückzuführen ist und eine Tatsachenbehauptung, die rechtlich angreifbar ist, dann nicht anzunehmen ist, wenn sie lediglich für die Beurteilung und für die daraus folgende Meinungsäußerung Grundlage war. Bereits ein Vergleich ist eine Schlußfolgerung aus Beobachtungen und Uberlegungen und somit im Sinne der Rechtssprechung ein Werturteil. 
Solange im Meinungsäußerungsrecht nicht in den Wirtschaftskampf eingetreten wird, ist es durchaus erlaubt, zu vergleichen, auch wenn dadurch der Gegner herabgesetzt wird. Ein solcher rein meinungsmäßiger Vergleich ist erlaubt, solange nicht in einen Gewerbebetrieb eingegriffen wird.

Diese Aussagen erscheinen ein wesentliches Kriterium für kirchliche Medienarbeit zu sein, insbesondere im Hinblick auf Aussagen von der Kanzel her (wenngleich dieser Beitrag sich speziell noch nicht mit der Problematik von sog. Wahlhirtenbriefen befaßt hat).

\section{Anmerkungen:}

1. $\{2$ Reichspressegesetz: Das gegenwärtige Gesetz findet Anwendung auf alle Erzeugnisse der Buchdruckerpresse sowie auf alle anderen durch mechanische oder chemische Mittel bewirkten zur Verbreitung bestimmten Vervielfältigungen von Schriften..., $\ 6$ Bayerisches Pressegesetz: Druckwerke im Sinne dieses Gesetzes sind alle mittels der Buchdrudkerpresse oder eines sonstigen Vervielfältigungsverfahrens hergestellten ... Schriften .... usw.

2. NJW 69, Seite $744 \mathrm{ff}$.

3. BVerfGE 7, 198.

4. BVerfGE $10,118$.

5. BVerfGE $5,88$.

6. BVerfGE 12, 113.

7. BGH a.a.O.

8. Art. 5 Abs. 1 Satz 1 GG.

9. Von Mangold-Klein, Anm. III, 2 zu Art. 5 GG.

10. BVerfGE 5, 134.

11. BVerfGE 7, 189.

12. BVerfGE $12,113$.

13. BGH Z 45, 296/308.

14. Vgl. BVerfGE 7,$198 ; 10,118 ; 20,162$.

15. Vgl. BGH GRUR $68,317$.

16. A. a. O.

17. BVerfGE 21, 278.

18. BVerfGE 7, 99; 14, 121.

19. Vgl. $\ 11$ Reichpressegesetz; $₫ 10$ Bayerisches Pressegesetz; $\ 10$ hess. Pressegesetz; $₫ 11$ Baden-Württemb. Pressegesetz; $\ 10$ Berlin; $\$ 11$ Bremen; $\ 11$ Hamburg; $\$ 11$ Niedersachsen; $\ 11$ Nordrhein-Westfalen; $\ 11$ Rheinland-Pfalz; $\ 11$ Saarland; $\ 11$ SchleswigHolstein.

20. OLG Hamburg NJW 67, 159.

21. BGH in NJW 65, 1476 NJW 65, 294 NJW 62, 152.

22. NJW $54,1252$.

23. NJW 59, 636.

24. NJW $67,159$.

\section{S U M MAR Y}

Some aspects of church media work in the framework of secular law are presented since also dhurch media work cannot escape the juridical stand before secular law. The limitations towards media work given by the law in the Federal Republic of Germany are also valid for the communications work of the Church. In what cases are there e.g. certain juridical conditions that priests should be aware when they go to the pulpit? There are certainly juridical aspects in church communications which have to be recognized. 


\section{RESUMÉ}

L'auteur montre certains aspects du travail de l'Eglise dans le domaine des mass media soumis au droit séculier: afin que le travail de l'Eglise dans le domaine des mass media soit considéré dans le droit séculier comme tout autre travail des mass media. En République Fédérale d'Allemagne, les limites du droit commun valent aussi bien pour le travail de l'Eglise avec les mass media que pour toute autre institution s'occupant de mass media. Il fait voir aussi dans quel cas de valeur philosophique une nouvelle marge est donnée, surtout en considération des témoignages prononcés du haut de la chaire. Il en résulte pour le travail de l'Eglise dans le domaine des mass media un critère juridique essentiel dont il faut tenir compte.

\section{RESUMEN}

El autor analiza aspectos de los medios de difusión eclesiales en el contexto del derecho secular: preconiza que el trabajo de difusión eclesial sea considerado por el derecho secular como cualquier otra actividad de difusión. Ios límites del derecho general en la República Federal de Alemania tienen vigencia tanto para los medios de difusión de la Iglesia como para cualquier otro órgano de comunicación social. Con una valoración ideológica muestra además en qué caso existe un margen mayor, sobre todo con vistas a exposiciones desde el púlpito. 